\title{
THE KINKED INNOMINATE VEIN
}

\author{
BY \\ K. SHIRLEY SMITH \\ From the Cardiac Department, Charing Cross Hospital \\ Received July 1, 1959
}

The examination of the neck is an essential part of every investigation of the heart. Over-filling of cervical veins had long been known as a sign of heart failure when Lewis (1930) laid down standard methods for this test, which needs attention to detail and close scrutiny; even so, the appraisal is sometimes difficult. The meaning of fixed bilateral filling as compared with ebb and flow is selfevident, but unilateral venous congestion, when there is no clear causal obstruction is not so easy to understand. In this paper, attention is drawn to the frequent occurrence of unilateral filling of the left external jugular vein and reasons are given for regarding it as a sign of a high and probably rigid aortic arch. Since this sign is caused by the aorta compressing the left innominate vein from below, the sign is named the kinked innominate vein.

A high aortic arch frequently causes tortuosity of the right common carotid artery which winds below the clavicular head of the sternomastoid muscle. This physical sign was first fully described by Parkinson et al. (1939) who studied it in 47 patients and named it the "kinked carotid". They found that it was generally associated with hypertension and arteriosclerosis, but in one-quarter of the cases with arteriosclerosis alone. It occurred in women but hardly ever in men. The new sign that is now defined is in a sense a natural counterpart of the arterial sign, both being due to the same cause, namely, a high and probably rigid aortic arch. The signs have in common also the fact that they may lead to mis-diagnosis-on the one hand, a false diagnosis of aneurysm of the carotid or innominate artery, and on the other a suspicion of glandular or other cause of obstruction in the mediastinum or even of heart failure. The differential diagnosis will be considered in more detail later.

\section{Clinical Features}

In the patient of medium or spare build, lying relaxed at an inclination of $45^{\circ}$, some normal structures of the neck are clearly seen. They are shown on the right side of the neck in Fig. 1 and 2. In both of these photographs the sternal and clavicular heads of the sterno-mastoid stand out and the lateral border of the clavicular head makes an angle of about $90^{\circ}$ with the clavicle. Superficial veins may be seen but they are not filled, as may be shown by the usual clinical test. In the normal person these land-marks are seen on both sides of the neck, hardly any disparity being noticeable.

In order to elicit the sign of the kinked innominate vein, the observer must view the patient as he lies relaxed with the trunk at an inclination of $45^{\circ}$. He must inspect the neck in a good light either from the foot of the couch or by leaning well across the patient, who must not move his head. These apparently obvious points are emphasized, because a helpful patient often unwittingly obscures the sign by turning his head a little to the right as the observer leans across to view the left side of the neck. This may be the reason why a search has failed to reveal any previous record of this sign. In Fig. 1 it is seen that the usual clear border of the sterno-mastoid is broken on the left side by the filled external jugular vein which forms a slightly sigmoid prominence in the left 

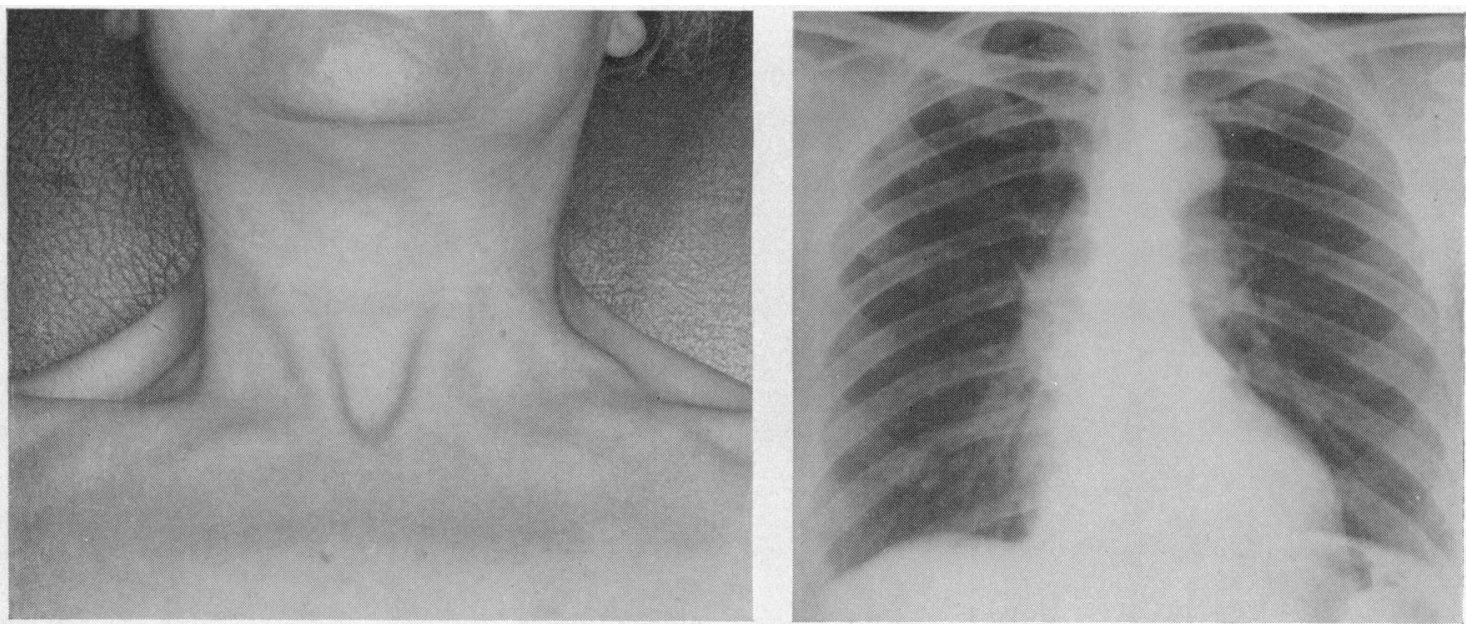

FIG. 1.-Woman, aged 56. B.P. 210/130. No dyspnœea; not in heart failure. Radioscopy shows left ventricular hypertrophy. X-ray of chest shows high and unfolded aortic arch. The distended left external jugular vein crosses the sterno-mastoid muscle to lie in the floor of the posterior triangle of the neck.
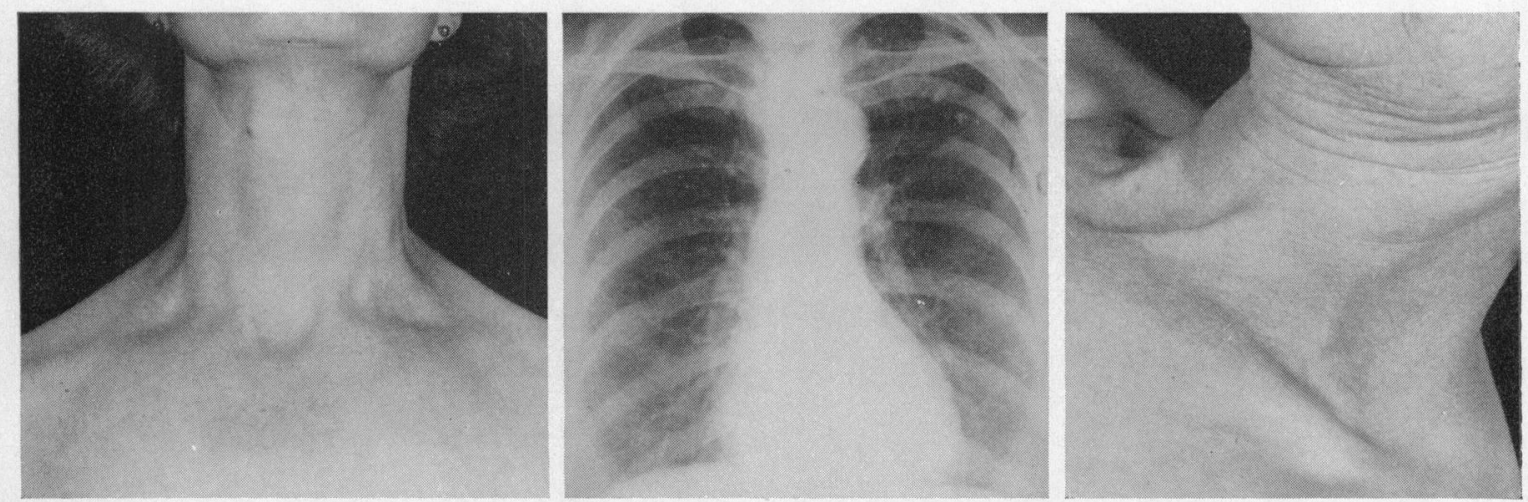

Fig. 2.-Woman, aged 61. B.P. 260/130. Advanced arteriosclerosis; not in heart failure. Suprasternal pulsation in neck. X-ray shows high and atheromatous aortic arch. The over-filled external jugular vein lies in the floor of the posterior triangle.

posterior triangle. A similar appearance is seen in the left side of the neck in Fig. 2. Sometimes there is a most conspicuous disparity between the veins on the two sides, as shown in Fig. 3 . Kyphosis may contribute to the sign by, as it were, lowering the great veins on to an aortic arch already high, as in Fig. 4. Fig. 5 shows, as does Fig. 4, the two sides of the neck photographed with suitable and identical lighting and in both of these instances the sign of the kinked innominate is plainly shown. The ages of the patients ranged from 50 to 66 .

\section{ANATOMY AND CAUSATION}

The sign of the kinked innominate has been observed by me in scores of patients since I first took note of it in 1937. The difficulties of putting it on pictorial record, and especially in patients seen in the consulting room are obvious. However, the five examples here described and photographed suffice to make clear its features as already detailed.

Anatomy. The left subclavian and the left jugular veins unite at the level of and posterior to the left sterno-clavicular joint to form the left innominate vein. Measurements of the size of the 

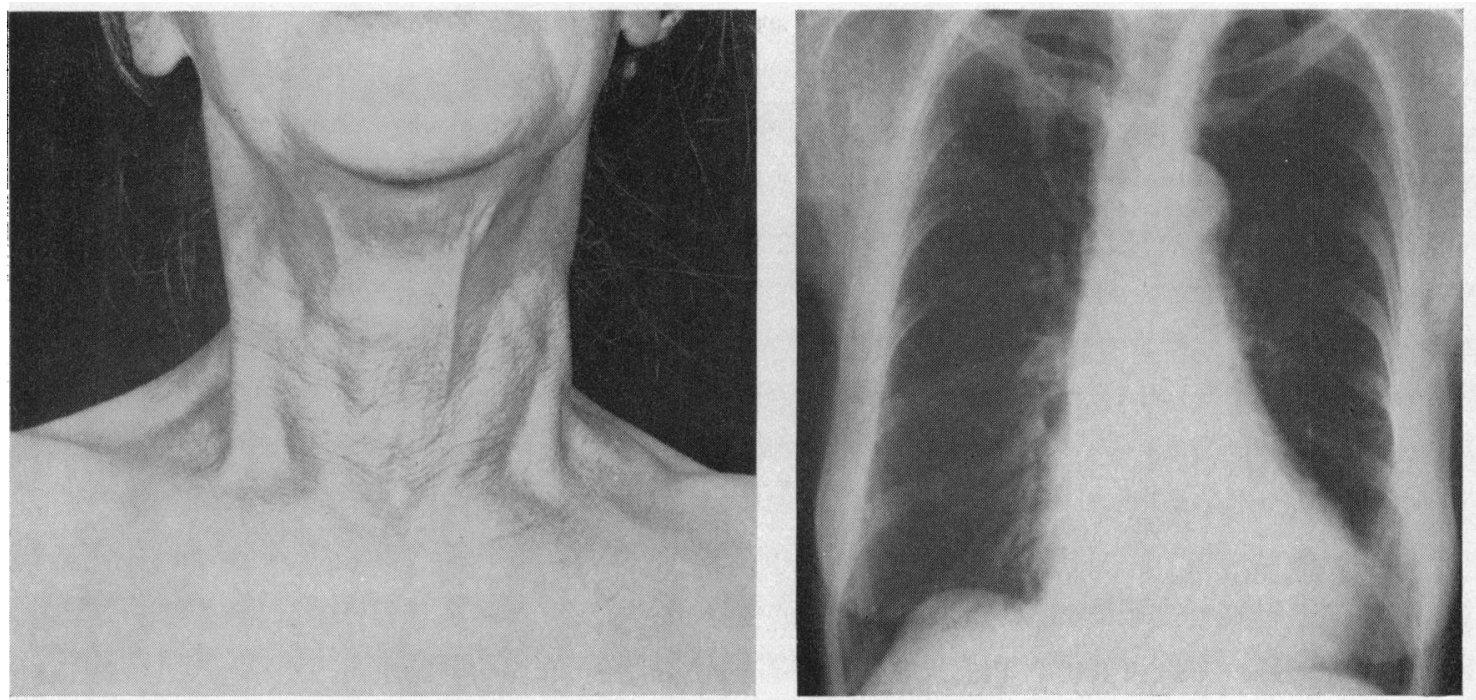

FIG. 3.-Woman, aged 66. Cardiac infarction; 2:1 A-V block; later, auricular fibrillation, but no signs of heart failure. B.P. 170/105. X-ray shows enlarged left ventricle and an elongated atheromatous aorta. External jugular vein breaks the line of the sternomastoid muscle and ends in a flat prominence in the floor of the posterior triangle.
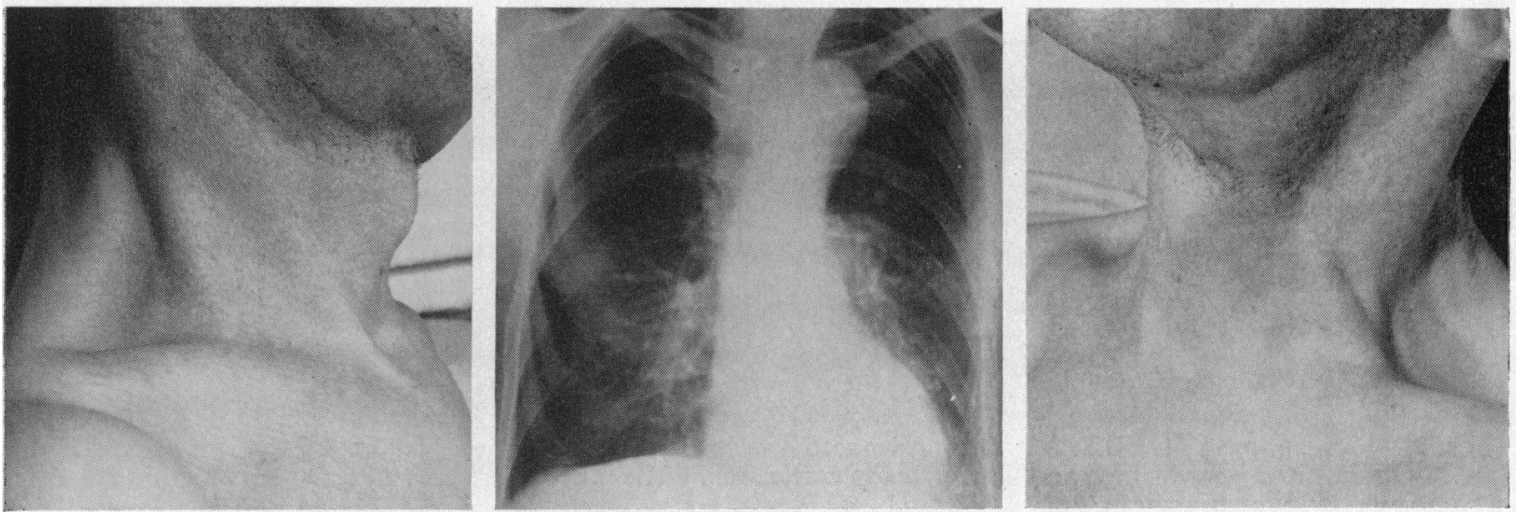

FIG. 4.-Man, aged 60. Admitted with cerebral thrombosis and left hemiplegia. B.P. 230/140. Generalized arteriosclerosis. Kyphoscoliosis exaggerates height of aortic arch seen in X-ray, which also shows left ventricular enlargement. Conspicuous disparity of the external jugular veins.

normal left innominate vein have been made by Roberts et al. (1951) by means of angiography. Among 66 patients (average age 48 years) they found the mean length to be $7 \cdot 2 \mathrm{~cm}$.; the diameter ranged from 5 to $18 \mathrm{~mm}$, with a mean of $11.7 \mathrm{~mm}$. This vessel passes to the right and slightly downward to join with the right innominate vein in forming the superior vena cava. In its course the left innominate vein comes into relation at its lower border with the antero-superior surface of the arch of the aorta (Fig. 6). Springing from the latter are the great trunks of the left common carotid, left subclavian and the innominate arteries; at their origins, these vessels are posterior relations of the left innominate vein (Fig. 7).

High Aortic Arch, Arteriosclerosis, and Atheroma. Bearing in mind the anatomical features just described, it will readily be understood that if the aortic arch rises higher than its usual position it will compress the vein from below. As Hinshaw and Rutledge (1942) have pointed out, "these 

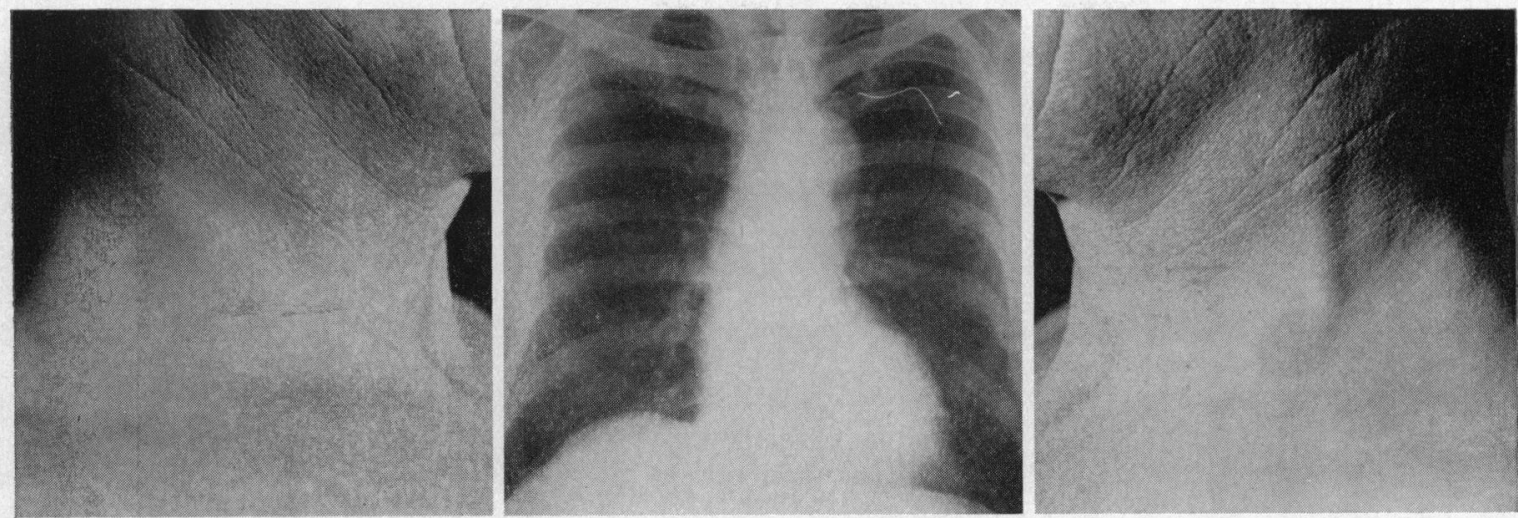

FIG. 5.-Man, aged, 57. Angina of effort. Essential hypertension. B.P. 265/160. Not in heart failure. X-ray shows left ventricular hypertrophy and a high unfolded aortic arch, which causes the overfilling of the cervical veins on left side.

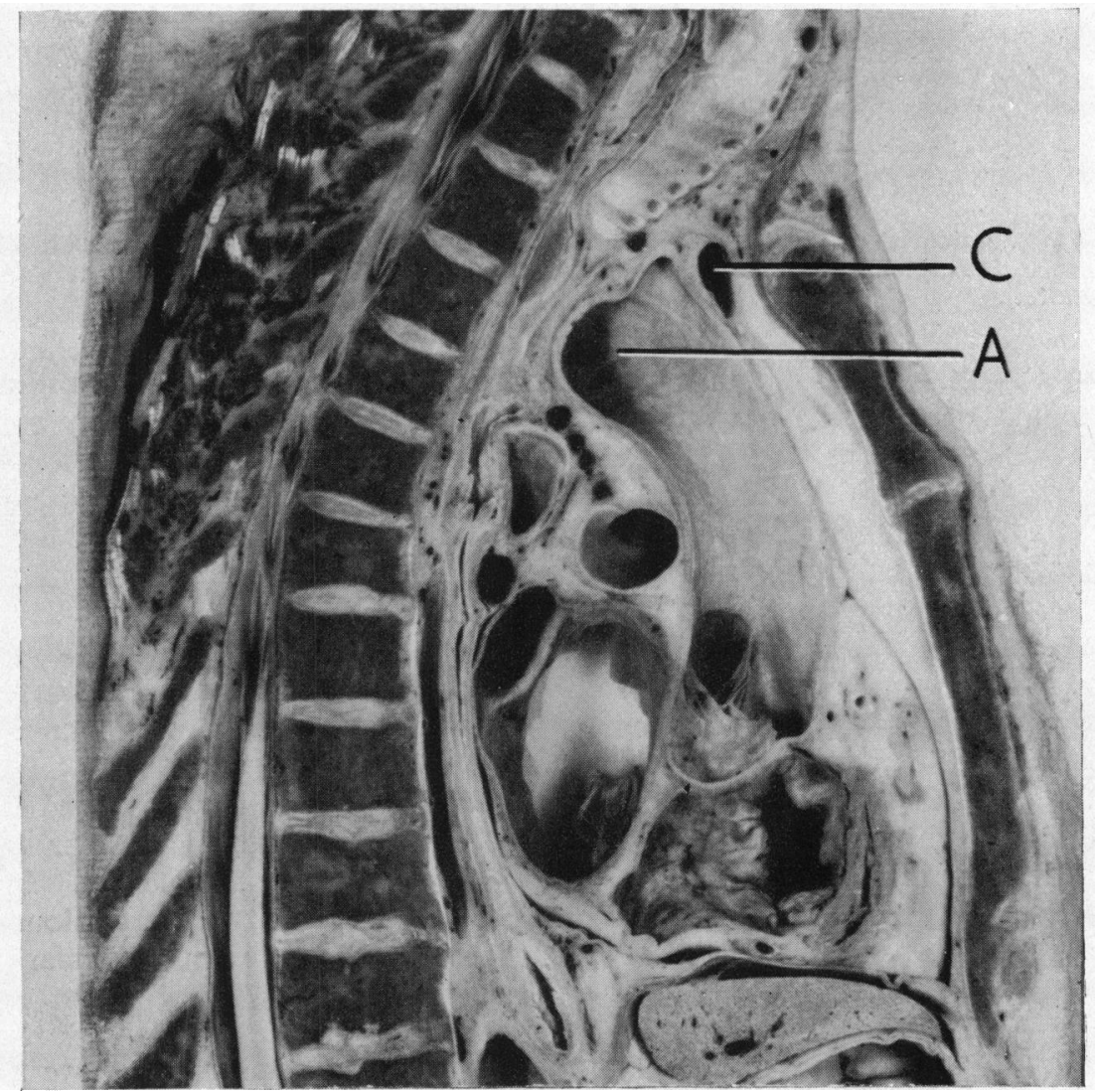

Fig. 6. - Vertical section of thorax to show normal relation of aortic arch (A) to left innominate vein (C). The vein is in a position in which it could readily be compressed between an enlarged aortic arch and the manubrium sterni. 


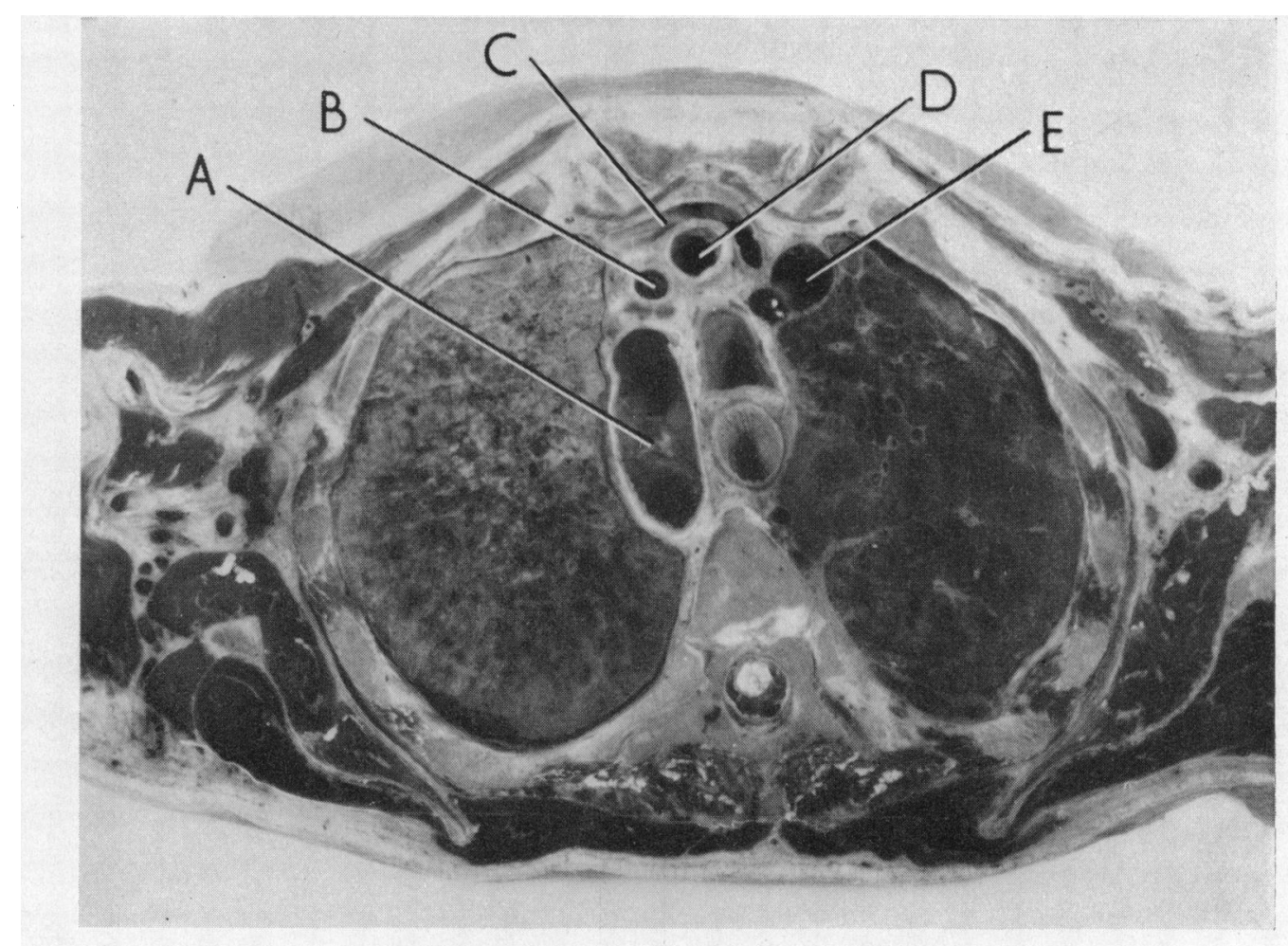

FIG. 7.-Horizontal section of thorax at level of left innominate vein (C) to show normal relation between it and innominate artery (D), left common carotid (B), right innominate vein (E), and aorta (A).

thin-walled veins in the mediastinum are filled with blood under very low pressure. They are anteriorly situated close to the bony thorax and may consequently be readily compressed by expanding mediastinal lesions". Equally, any enlarging structure, such as the aortic arch, may compress them. Conditions that are known to displace the arch upward are high blood pressure, which also unfolds the arch of the aorta, and arteriosclerosis which causes elongation of the vessel even in the absence of hypertension. Similar changes in the great trunks that spring from the arch behind the vein may contribute to the compression.

Atheroma of the Aorta. A rigid aortic arch, as is often found with atheroma and calcification, will be likely to interfere more with neighbouring venous flow than would an aorta dilated dynamically and therefore exerting only intermittent pressure.

In all of the instances here quoted and illustrated an X-ray film of the chest has been taken and some are reproduced with the photographs of the neck. In every case the aortic arch is seen to be abnormally high, and in two patients (Fig. 2 and 3) is obviously atheromatous. In those three cases in which it is not, the aorta shows the unfolding characteristic of hypertension. In none of the X-rays is there anything to suggest a local cause of venous obstruction, such as enlarged lymphatic glands or a retrosternal goitre.

Hypertension. Nearly all persons showing the kinked innominate sign have hypertension. The blood pressures of the five patients illustrated were: $260 / 130,265 / 160,210 / 130,230 / 140$, and $170 / 105 \mathrm{~mm}$. Hg. The influence of hypertension in elevating and unfolding the arch of the aorta has already been referred to. 
Possible Local Causes. It might be questioned whether the filling of the left external jugular vein could be due to local causes, for example compression by cervical fascia. If this were so there should be no disparity in the venous pressure in the arms in patients showing this sign. To test this possibility venous pressures have been recorded by saline manometry in the antecubital veins in several patients showing this sign. The results of these observations are given in Table I.

TABLE I

Comparative Brachial Venous Pressures in Patients with Kinked Left Innominate Vein

\begin{tabular}{|c|c|c|c|c|c|}
\hline \multirow[t]{2}{*}{ Patient } & \multirow{2}{*}{\multicolumn{2}{|c|}{ Age and sex }} & \multirow[t]{2}{*}{ B.P. } & \multicolumn{2}{|c|}{$\begin{array}{l}\text { Venous pressures in } \mathrm{cm} \text {. water } \\
\text { related to sternal angle }\end{array}$} \\
\hline & & & & Rt. antecubital & Lt. antecubital \\
\hline $\begin{array}{l}\text { R.C. } \\
\text { H. } \\
\text { O. } \\
\text { M.B. }\end{array}$ & $\begin{array}{l}44 \\
53 \\
51 \\
52\end{array}$ & $\begin{array}{l}\text { M } \\
\text { F } \\
\text { F } \\
\text { F }\end{array}$ & $\begin{array}{l}220 / 140 \\
220 / 140 \\
210 / 110 \\
160 / 100\end{array}$ & $\begin{array}{c}0.3 \\
\pm 0 \\
0.3 \\
\pm 0\end{array}$ & $\begin{array}{c}3.5 \\
12 \\
7 \cdot 5 \\
2 \cdot 7\end{array}$ \\
\hline
\end{tabular}

These results suggest that when the sign is present there is a rise of pressure in the whole of the venous system of the neck and arm on the left side: the disparity must therefore be due to compression proximal to the junction of the subclavian and innominate veins. It has already been shown that compression by a high aortic arch is a reasonable and even probable explanation.

There remains the possibility that the pressures in the brachial veins are naturally unequal. Measurements in a number of control subjects have shown that this is not the case (Table II).

TABLE II

Comparative Brachial Venous Pressures in Normal Controls

\begin{tabular}{c|c|c|c|c|c|c|c|c|c|c|c|c|c}
\hline & \multicolumn{7}{|c|}{ Male subjects } & \multicolumn{5}{c}{ Female subjects } \\
\hline Control & 1 & 2 & 3 & 4 & 5 & 6 & 7 & 1 & 2 & 3 & 4 & 5 & 6 \\
\hline $\begin{array}{c}\text { Pressure in R. ante- } \\
\text { cubital vein }\end{array}$ & 4.9 & 2 & \pm 0 & \pm 0 & \pm 0 & \pm 0 & \pm 0 & \pm 0 & $2 \cdot 3$ & \pm 0 & \pm 0 & \pm 0 & 0.2 \\
\hline $\begin{array}{c}\text { Pressure in L. ante- } \\
\text { cubital vein }\end{array}$ & \pm 0 & \pm 0 & \pm 0 & 0.3 & 0.5 & 0.2 & $2 \cdot 1$ & 0.8 & \pm 0 & 0.1 & 0.4 & 0.3 & \pm 0 \\
\hline
\end{tabular}

Venous pressure in $\mathrm{cm}$. water related to sternal angle.

\section{The Diagnostic Significance of The Sign}

The clinical diagnosis of enlargement or elongation of the aorta may be obvious at first sight, as when an aorta increased in girth produces a general thrust or a local pulsation visible and palpable at the upper part of the chest. Likewise, an aortic arch rising very high may produce a pulsation palpable in the suprasternal notch. Beyond these signs there are few that are dependable. Lewis (1946) claimed that central dullness on percussion across the second and sometimes the first intercostal space was a valuable sign, but at best it is inconclusive. An aortic second sound that is loud and sometimes palpable ("diastolic shock") suggests an increased girth or rigidity of the ascending aorta, but again, this cannot be regarded as a first-class sign. The kinked carotid is a valuable sign of a high aortic arch but it is almost confined to women. In the circumstances, the value of the new sign becomes clear in that it provides an additional and important piece of evidence indicating a high and probably rigid aorta. Moreover, it is found as often in men as in women. What is the explanation of this differing sex incidence of the kinked carotid and the kinked innominate is not known. 
It should be emphasized that this sign has nothing to do with the cervical venous signs found in congestive heart failure, although this may be suggested at first glance. In this state, with failure of the right ventricle or the heart as a whole, the cervical veins tend to be more in evidence on the right than the left (Hunter, 1927). Of the patients here described, none was in heart failure. The X-rays of the chest show no hilar congestion or pulmonary plethora, with the possible exception of the patient in Fig. 4 whose state was deteriorating through cerebral thrombosis-but it will be seen that in him the right side of his neck shows no venous congestion, and the film gave abundant explanation of the unilateral venous congestion.

There are several other conditions that may cause appearances simulating this sign. Obstruction by neoplasm is the commonest but it is likely to cause general obstruction of the superior veha caval system, whether the tumour be benign (e.g. goitre) or malignant and whether the mass be primary or of secondary glandular origin. Operative procedures such as thoracoplasty and pneumonectomy may interfere with drainage of the superior vena caval system but, again, this is more likely to cause bilateral or right-sided signs than solitary left-sided congestion. Exceptions to this generalization are quoted by Norris and Landis (1938).

\section{SUMMARY}

A study has been made of unilateral left-sided engorgement of the external jugular vein. It is found to be produced by pressure upon the left innominate vein exerted from below by a high and probably rigid (atherosclerotic) arch of the aorta.

This sign is analogous to the well-known kinked carotid but unlike that sign it is seen as often in men as in women. It has nothing to do with the venous congestion of heart failure which is bilateral.

Photographs of the sign are shown and corresponding X-ray films, demonstrating the relationship to a high and sometimes atheromatous aorta.

Hypertension, essential or atherosclerotic, of moderate or severe grade is almost always present when this sign is seen.

Measurements of brachial venous pressure have shown materially higher pressures on the left side in patients with this sign. On the other hand, such comparisons in normal controls have shown that ordinarily the venous pressures in the arms are practically equal.

The kinked innominate is held to be a useful addition to the rather tenuous means hitherto available for the clinical diagnosis of aortic enlargement.

I have to thank Dr. J. N. Mickerson for much technical assistance. To Miss P. M. Turnbull, Medical Photographer to the Charing Cross Hospital, I am indebted for the means of clearly depicting this sign.

\section{REFERENCES}

Hinshaw, H. C., and Rutledge, D. I. (1942). J. clin. Lab. Med., 27, 908.

Hunter, D. (1927). Proc. Roy. Soc. Med., 20, 336.

Lewis, T. (1930). Brit. med. J., 1, 849.

- (1946). Diseases of the Heart. p. 219, London.

Norris, G. W., and Landis, H. R. M. (1938). Diseases of the Chest and the Principles of Physical Signs. p. 960. London.

Parkinson, J., Bedford, D. E., and Almond, S. (1939). Brit. Heart J., 1, 345.

Roberts, J. D., Dotter, C. T., and Steinberg, I. (1951). Amer. J. Roentgen. Radium Therapy, 66, 341. 